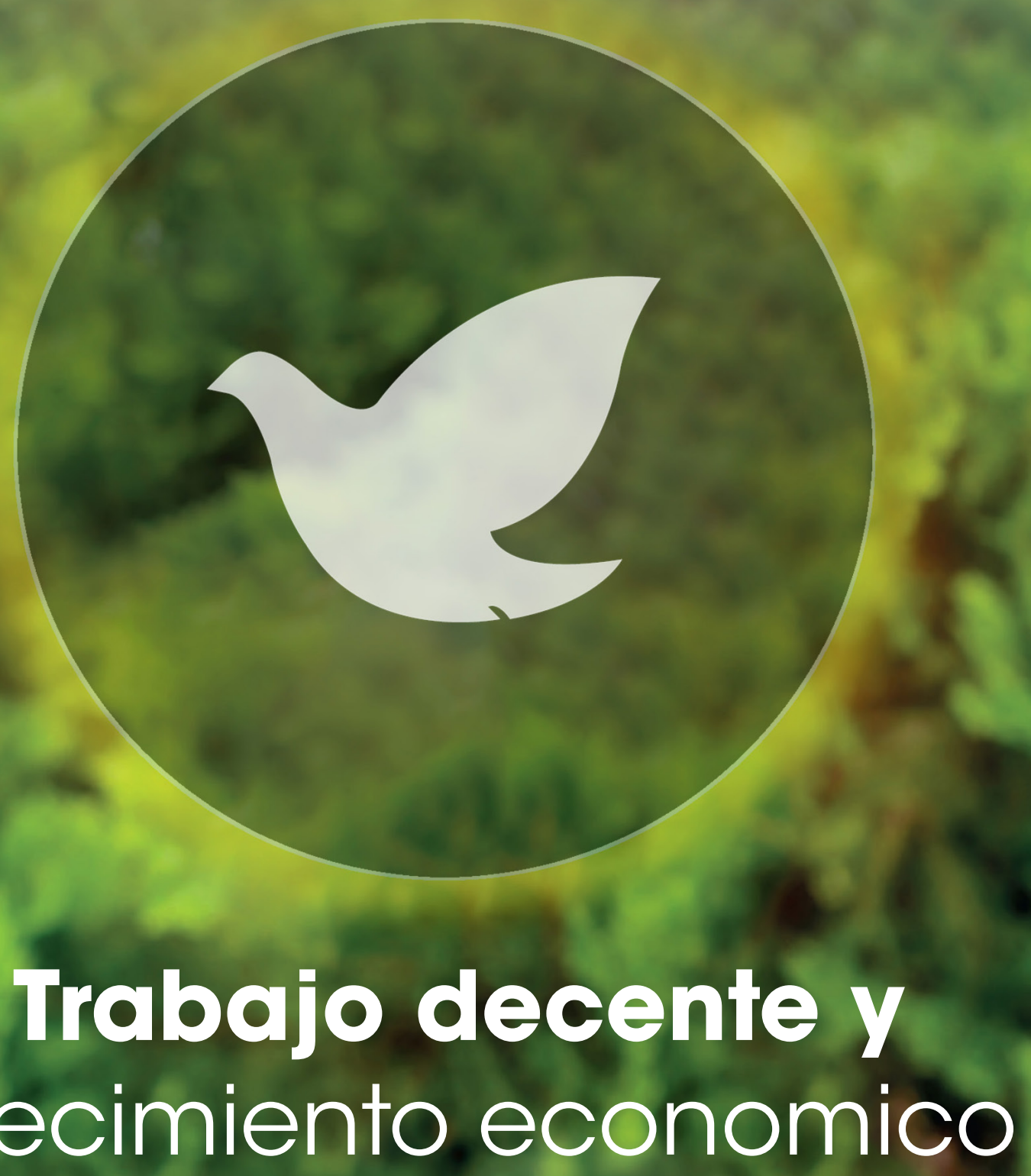




\section{DISEÑO DE UNA CARTILLA EN EDUCACIÓN FINANCIERA PARA LAS MICROEMPRESAS \\ DE LA LOCALIDAD ANTONIO NARIÑO \\ (BOGOTÁ D.C)}

Oscar Fabián Daza Ardila Jeisson Arango Ramos Kimberly Dayana Caro

Nataly Hernández Serna ${ }^{19}$ Jenny Alexandra Segura Osuna20

\section{Corporación Universitaria Minuto de Dios}

\section{Resumen:}

En la localidad Antonio Nariño (Bogotá D.C) se está trabajando en el diseño de una cartilla en educación financiera con el fin de informar a las micro, pequeñas y medianas empresas (Mipymes) en temas que influyen en su actividad comercial; tanto en el sector educativo como en el financiero de esta comunidad. Dicho material tiene como objetivo instruir a estos tipos de empresa con las diversas opciones de financiamiento que encuentran: proveedores, clientes, socios, créditos y bancos; ya que en su mayoría tienen dificultad para acceder a estas fuentes. Adicionalmente, muchas de estas empresas no se conforman de manera formal, lo que ocasiona una debilidad en su crecimiento y baja competitividad con respecto a las que sí acceden a ellas. Por estas razones, se emplean opciones de manejo por medio del campo financiero en presupuestos, sistemas de ahorro, créditos y microcréditos de inclusión; así mismo, se trazan temas como el manejo del dinero y la protección a la que el consumidor puede acceder y que por lo general desconocen por falta de conocimiento herramientas, siendo una de las principales causas de que las Mipymes de esta localidad no puedan avanzar

\section{Palabras clave:}

educación financiera; inclusión financiera; empresas; financiación.

\footnotetext{
${ }^{19}$ Estudiantes en el programa de administración de empresas. Integrantes del semillero de investigación Administrarte. Correos electrónicos: odazaardila@uniminuto.edu.co, jarangoramo@uniminuto.edu.co, Kimberly. caro@uniminuto.edu.co, Nataly.hernandez@uniminuto.edu.co

${ }^{20}$ Docente de investigación. Correo electrónico: jseguraosun@uniminuto.edu.co
} 


\section{Abstract:}

Antonio Nariño (Bogotá D.C) is working on the design of a booklet in financial literacy in order to inform micro, small and medium enterprises (MSMEs) on issues that influence their commercial activity; both in the educational and financial sectors of this community. This material aims to instruct these types of companies with the various financing options they find: suppliers, customers, partners, credits and banks; since they mostly have difficulty accessing these sources. Additionally, many of these companies do not conform formally, which causes a weakness in their growth and low competitiveness with respect to those that do access them. For these reasons, management options are used through the financial field in budgets, savings systems, credits and inclusion microcredits; likewise, issues such as money management and the protection that consumers can access and which are generally unaware of lack of knowledge tools are outlined, being one of the main causes that MSMEs in this town cannot advance.

\section{Keywords:}

financial literacy; Financial Inclusion; My SME's; Financing.

\section{Introducción}

La educación financiera es una herramienta a través de la cual diferentes personas desarrollan habilidades y actitudes que les permiten organizar y gestionar sus recursos económicos, lo que contribuye a una buena toma de decisiones financieras, para mejorar su bienestar económico (OECD, 2005).

En este sentido, a través de la realización de una cartilla en educación financiera como medio de difusión de conocimiento dirigida a las Mipymes de la localidad Antonio Nariño en Bogotá D.C; se espera promover herramientas prácticas que permitan conocer y mejorar el uso de sus recursos. Dentro de los aportes se espera: difundir conceptos básicos que influyen para bien en la toma 
de decisiones de las empresas, creando estrategias que faciliten el desempeño en la gestión del dinero; que a su vez les permita seguir activas y productivas en el mercado, aportando al bienestar no solo de la localidad y la ciudad; sino, del país en general.

\section{Metodología}

Se plantea un enfoque de tipo mixto, empezando por un estudio explicativo para exponer el grado de educación financiera como variable importante que influye en los resultados económicos y estabilidad de las Mipymes; estableciendo relaciones de causa y efecto, el proceso formal. El método que se emplea en el estudio es deductivo; es decir, parte de una idea general, para obtener unas conclusiones de un caso particular. Así mismo, parte de la revisión de literatura internacional y local. La naturaleza de los datos se efectúa en dos tipos de metodología, cuantitativa y cualitativa; empleando datos estadísticos y un estudio descriptivo, desarrollando por una parte encuestas en la localidad de Antonio Nariño donde se establecieron los temas que iban dirigidos a las MIPYMES y por otra, un análisis subjetivo e individual de los participantes, mostrando así los resultados que se verán a continuación.

Según las estadísticas de la Cámara de Comercio de Bogotá al 2018, existían 7.032 empresas con registro en esa entidad; de las cuales el 92.16\% son microempresas, el $6.61 \%$ son pequeñas empresas, el 1.12\% son medianas empresas y tan solo el $0.1 \%$ grandes empresas. De este modo, las encuestas del proyecto se aplicaron a 50 empresas de la localidad, número elegido a conveniencia de acuerdo con los objetivos de dicha investigación; en su mayoría a microempresas, dado su peso respecto al total de unidades económicas de la localidad. A partir de los resultados obtenidos en el trabajo de campo junto con la revisión bibliográfica se eligieron los temas más relevantes a ser incluidos en la cartilla. 


\section{Resultados y discusión}

Hoy en día las empresas están luchando por tener una mayor participación en el mercado, para lograr esto es necesario un crecimiento constante, aunque por las circunstancias que se presentan estos microempresarios se ven obligados a manejar sus recursos financieros de manera "inadecuada". Dado lo anterior, la localidad Antonio Nariño no es indiferente al tema ya que las Mipymes que allí funcionan tienen un panorama desafiante, debido a la dinámica que manejan han desarrollado un ambiente cada día más inestable y complejo por diversos factores como lo son la competitividad, globalización y su poco reconocimiento en el mercado. Para solventar estas desfavorables debilidades, es necesario brindar un conocimiento en educación financiera capaz de mitigar riesgos, dar soluciones y planeaciones asertivos con el fin de proveer su permanencia y crecimiento en el mercado; por lo cual surge un plan de diseño en una herramienta de difusión sobre la educación financiera para las microempresas de esta localidad.

De acuerdo con lo anterior, en este proyecto de investigación se plantea la siguiente pregunta: ¿Cuál es el grado de educación financiera que tienen las microempresas de la localidad de Antonio Nariño? En este sentido, al finalizar el estudio se espera brindar una herramienta de capacitación en esta materia a través de una cartilla.

Si se habla de personas o de empresas, la falta de educación financiera es un problema grave que de acuerdo con estudios empíricos se relacionan con el nivel socioeconómico, género, edad, o nivel de estudios (Lusardi, 2008; Rodríguez-Parra y Acosta-Triviño, 2017). Dado lo anterior, la educación financiera es un factor importante para la sociedad y el progreso económico de un país. Sin embargo, en Colombia y otros países se evidencia que hay un problema de exclusión financiera (Pavón, 2016), que afecta a las personas más vulnerables que suelen ser los de menores recursos y/o con niveles de educación bajos. 
Dentro de la presente investigación se ha encontrado que el tema de la educación financiera para la supervivencia de las empresas es muy importante como lo señala Asobancaria (2017), en temas como el dar a conocer a las Mipymes las opciones de financiamiento que se encuentran disponibles en el mercado formal: los proveedores, clientes, socios, microcréditos y bancos, ya que en su mayoría tienen dificultad para acceder a estas fuentes por diversos motivos como que no cuentan con los requisitos suficientes, adicionalmente muchas de estas no se conforman de manera formal, por lo que genera un problema al momento de obtener financiación de entidades del sistema financiero formal, ocasionando una debilidad en su crecimiento y baja competitividad con respecto a las que sí acceden a ellas. Zuleta (2017), muestra que en Colombia aún existe un alto grado de exclusión financiera en las empresas de menor tamaño, y que ello se deriva del desconocimiento de la estructura y los servicios del sistema financiero en el país.

De acuerdo con autores nacionales e internacionales se encuentra que los principales temas que tienen que ver con la educación financiera para empresas están relacionadas con los siguientes temas: sistemas de ahorro, créditos y microcréditos de inclusión, el manejo del dinero y la protección a la que el consumidor, presupuestos y riesgos financieros. A continuación, en la Figura 1, se presentan las temáticas a tener en cuenta a la hora de capacitar a los empresarios en temas de educación financiera. 
Figura 1. Revisión temática de la literatura
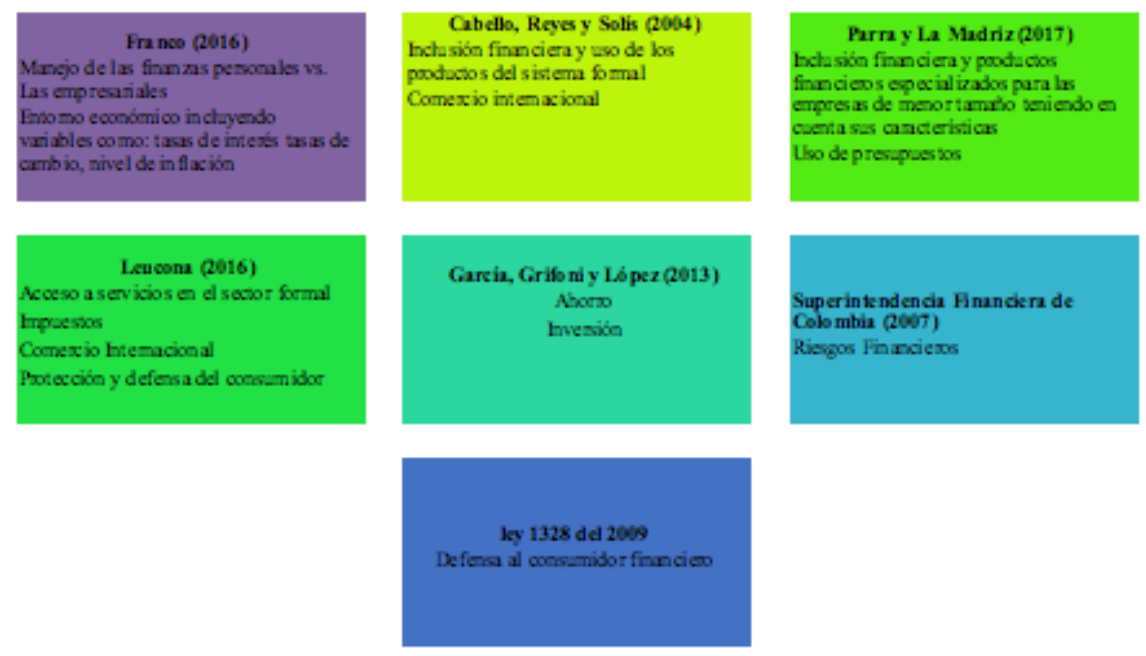

Fuente: elaboración propia

Con la aplicación de las encuestas se evidencia que en promedio los empresarios de la localidad Antonio Nariño se sienten menos preparados en temas como los referentes a la economía y, en general, el manejo de las finanzas empresariales. En cambio, perciben conocimientos suficientes en administración y mercadeo como se puede observar en la Figura 2:

Figura 2: Percepción del grado de educación financiera por parte de los empresarios de acuerdo a temáticas

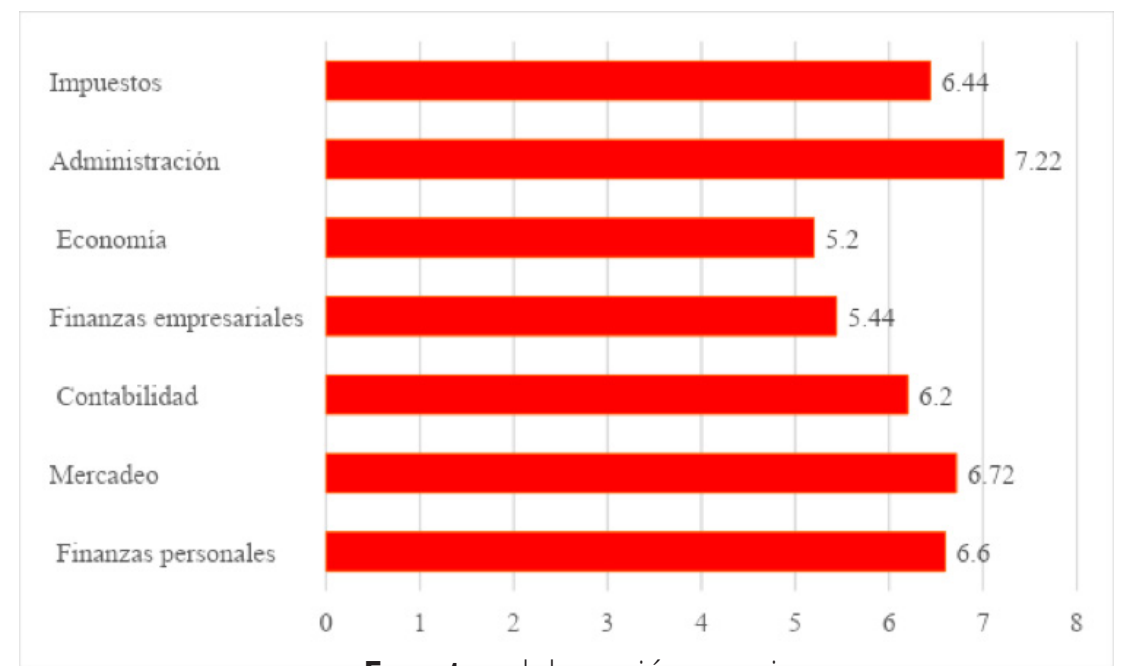

Fuente: elaboración propia 


\section{Conclusiones}

Teniendo en cuenta la participación de los empresarios en la producción nacional se sitúan las micro, pequeña y mediana industria, como motor de crecimiento económico y generación de empleos; por lo que es importante que ellas tengan conocimientos en educación financiera para optimizar las decisiones que toman y mejorar su bienestar. Hay que tener en cuenta que generalmente las Mipymes son el resultado de la iniciativa por alcanzar metas y logros, por parte de emprendedores con capacidad de innovar, pero no siempre están en la capacidad de desarrollar un plan de negocio donde se evalúe la estabilidad financiera que en el largo plazo se evidenciará en su permanencia en el mercado y hasta en su calidad de vida, este tipo de industria impacta positivamente la comunidad, estas empresas tienen un alto impacto en la economía del país, de manera positiva el entorno cultural, social y económico, respondiendo a este concepto global, en Colombia el Gobierno Nacional está buscando dentro del sistema de educación el desarrollo de una cultura de emprendimiento.

En cuanto a la generación de empleo este tipo de empresa genera puestos de trabajo en sectores socialmente débiles, de igual forma en la posibilidad de romper con la concentración de poder que caracteriza la economía colombiana que se ha visto golpeada por fenómenos sociales como el narcotráfico, violencia y altos niveles de pobreza.

En el caso específico de los empresarios encuestados, dueños o empleados con cargos administrativos de la localidad de Antonio Nariño en Bogotá se evidenció que hay falencias en el grado de educación financiera que tienen, por lo que una herramienta como la que se está construyendo sería un importante apoyo y fuente de conocimiento para ellos. 


\section{Referencias bibliográficas}

Asociación de Bancos e Instituciones Financieras de Colombia, Asobancaria. (2017). La educación financiera como motor de las MIPYMES en Colombia. Semana Económica 2017, 1094, 1-10. Recuperado de: https://www.asobancaria.com/wp-content/ uploads/2018/02/1094.pdf

Congreso de Colombia. (2009). Ley 1328 de 2009. Recuperado de: http://www. secretariasenado.gov.co/senado/basedoc/ ley_1328_2009.html

García, N., Grifoni, A. y López, J. (2013). La educación financiera en América Latina y El Caribe: Situación actual y perspectivas. Serie Políticas Públicas y Transformación productiva. Recuperado de: https://www.oecd.org/daf/ fin/financial-education/OECD_CAF_Financial_ Education_Latin_AmericaES.pdf

Lusardi, A. (2008). Financial literacy: an essential tool for informed consumer choice? (No. w14084). National Bureau of Economic Research, NBER. Recuperado de: https://www. nber.org/papers/w14084.pdf

Organización para la Cooperación y el Desarrollo Económicos, OCDE. (2005). Recomendación sobre los Principios y Buenas Prácticas de Educación y Concienciación Financiera. Recuperado de: https://www.oecd.org/daf/fin/ financial-education/[ES]\%20Recomendaci\%C3\%B3n\%20Principios\%20de\%20Educaci\%C3\%B3n\%20Financiera\%202005.pdf
Pavón, L. (2016). Inclusión financiera de las pymes en el Ecuador y México. Recuperado de: https://repositorio.cepal.org/ bitstream/handle/11362/40848/S1601083_ es.pdf?sequence $=1$ \&isAllowed $=y$

Rodríguez-Parra, P. y Acosta-Triviño, R. (2017). Delitos y conflicto en la localidad 16 Puente Aranda. Bogotá: Editorial Fundación Universitaria San Mateo. Recuperado de https://palma.sanmateo.edu.co/

Superintendencia Financiera de Colombia. (2007). Reglas relativas al sistema de administración de riesgo de mercado. Recuperado de: https://www.superfinanciera.gov.co/descargas?com=institucional\&name=pubFile1000189\&downloadname=cap21 riesgos_mercado.doc

Zuleta, L. (2017). Instrumentos para la inclusión financiera: el caso de Colombia. Recuperado de: https://www.cepal.org/sites/default/files/document/files/colombia_-_ instrumentos_watermark.pdf 